\title{
Computer Aid Screening of COVID19 using X-ray and CT Scan Images: A Comparative Study
}

\author{
Prabira Kumar Sethy * , Chanki Pandey ${ }^{\mathrm{b}}$, Santi Kumari Behera ${ }^{\mathrm{c}}$ \\ aDepartment of Electronics, Sambalpur University, Odisha, India \\ ${ }^{b}$ Department of Electronics and Telecommunication Engineering, Government Engineering College Jagdalpur, \\ CSVTU, CG, India

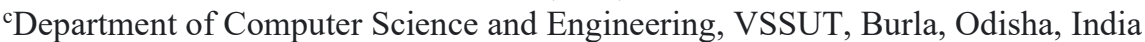 \\ Corresponding Author Email: prabirsethy.05@gmail.com \\ https://orcid.org/0000-0003-3477-6715
}

\begin{abstract}
In this article, we analyse the computer aid screening method of COVID19 using Xray and CT scan images. The main objective is to set an analytical closure about the computer aid screening of COVID19 among the X-ray image and CT scan image. The computer aid screening method includes deep feature extraction, transfer learning and traditional machine learning image classification approach. The deep feature extraction and transfer learning method considered 13 pre-trained CNN model. The machine learning approach includes three sets of features and three classifiers. The pre-trained CNN models are alexnet, googlenet, $\operatorname{vgg} 16, \operatorname{vgg} 19$, densenet201, resnet18, resnet50, resnet101, inceptionv3, inceptionresnetv2, xception, mobilenetv2 and shufflenet. The features and classifiers in machine learning approaches are GLCM, LBP, HOG and KNN, SVM, Naive bay's respectively. In addition, we also analyse the different paradigms of classifiers. In total, the comparative analysis is carried out in 65 classification models, i.e. 13 in deep feature extraction, 13 in transfer learning and 39 in machine learning approaches. Finally, all the classification models perform better in X-ray image set compare to CT scan image set.
\end{abstract}

Keywords: Computer-aided Screening; Coronavirus; X-Ray; CT scan; Machine Learning; Transfer Learning; Deep Learning.

\section{Introduction}

The COVID-19 also well knows as coronavirus is a deadly acute disease has appeared in Wuhan province, China in December 2019. COVID-19 outbreaks continue to spread over the world, over 18.6 million people across 200 countries [1]. At present COVID-19 pandemic is the most pressing issue confronting our whole world due to its massive negative impact on public health [2]. Although governments and rulers imposing various new schemes, policy and created new lifestyles to combat COVID-19. Science and technology have a major contribution in implementing these new schemes and policies [3].

On the other hand, recently artificial intelligence and computer-aided screening has been widely adopted and successfully applied for the acceleration of biomedical research and development [4]. Machine learning, deep learning and transfer learning have been used in many applications, including data classification [5] and image detection and segmentation [6]. There exist numerous open online databases, including chest X-Ray and CT scan datasets. This database is being used for building various classification and detection models and also can be used further too. Selecting an appropriate dataset out of the high amount of pandemic dataset is one of the challenging tasks. 
Recently, various studies have pointed out that X-Ray and CT images play an essential evaluation mean for screening and diagnosing COVID- 19 infection [7-21]. From the extensive review, it is found that using X-Ray dataset achieved results are quite significant and promising for COVID-19 diseases when compared with CT scan dataset. However, the main objective of this research is to provide a comparative analysis of various models of machine learning, deep learning and transfer learning used for diagnosis and detection of COVID-19 using X-Ray and CT scan images. In addition, it is required to know the limitation and understand the technological ability of image datasets so that the research push forward in a positive way to design computer aid diagnosis system for screening of COVID19.

\section{Data}

To build a consistent and robust model, it is essential to ensure the same number of samples while considering all the cases for each class. For this study, we have used the chest X-ray and Ct scan image available in Github and Kaggle repository. The publicly accessible database comprises of X-Ray and CT scans for both the class, i.e. normal images and COVID-19 infected images. The image samples involved in this comparative analysis is detailed in Table 1.

Table 1 The distribution of samples

\begin{tabular}{lll}
\hline Class & Type of Dataset images & No. of samples \\
\hline Normal & X-Ray & 350 \\
& CT Scan & 350 \\
\hline COVID-19 & X-Ray & 350 \\
& CT Scan & 350 \\
\hline
\end{tabular}

\section{Methods and Experimental Results}

In this section, we provide a comparative analysis of various models of machine learning, deep learning and transfer learning approach using X-Ray and CT scan images for the screening of COVID19. The exploration study was actualised utilising the MATLAB 2019a. All the applications were run on a laptop, i.e. HP Pavilion Core i5 5th Generation with basic NVIDIA GEFORCE GTX 1050Ti. The performance of each classifier is measured using six evaluation indicators namely accuracy, sensitivity, specificity, false positive rate (FPR), F1 Score and computational time in case of deep learning feature extraction and transfer learning approach. The accuracy and area under the curve (AUC) are used in the machine learning approach to measure the performance of classifiers. In deep feature extraction method, the most reliable classifier. Support vector machine (SVM) is employed to classify the COVID and NON-COVID images. The theory, mathematics and training parameter settings are as in our [18] research article.

\subsection{Deep Feature Extraction Approach}

Deep feature extraction is based on the extraction of features acquired from a pre-trained CNN. The deep features are extracted from fully connected layer and feed to the classifier for training purpose. Here, all the 13 pre-trained networks are considered for deep features extraction including alexnet, googlenet, vgg16, vgg19, densenet201, resnet18, resnet50, resnet101, inceptionv3, inceptionresnetv2, xception, mobilenetv2 and shufflenet. The deep 
features of pre-trained network fed to the SVM for classification. The classification model in deep feature extraction approach is illustrated in Figure1.

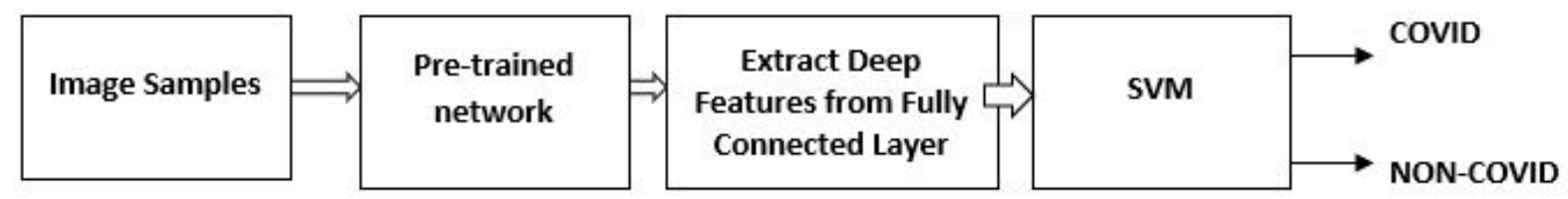

Figure1 Deep Feature Extraction Approach for Screening of COVID19.

After that, the classification is performed and evaluation indicators of all classification models are recorded in Table 2 .

Table 2. Comparative Results of Xray and CT Scan Images in Deep Feature Extraction with SVM Approach.

\begin{tabular}{|c|c|c|c|c|c|c|c|c|c|c|c|c|}
\hline \multirow{2}{*}{$\begin{array}{l}\text { Pre- } \\
\text { Train } \\
\text { ed } \\
\text { Mode } \\
1\end{array}$} & \multicolumn{6}{|c|}{ Xray Image } & \multicolumn{6}{|c|}{ CT Scan Image } \\
\hline & $\begin{array}{l}\text { Accura } \\
\text { cy }\end{array}$ & $\begin{array}{l}\text { Sensitiv } \\
\text { ity }\end{array}$ & $\begin{array}{l}\text { Specifi } \\
\text { city }\end{array}$ & FPR & $\begin{array}{l}\text { F1 } \\
\text { Score }\end{array}$ & $\begin{array}{l}\text { Comput } \\
\text { ational } \\
\text { Time }\end{array}$ & $\begin{array}{l}\text { Accur } \\
\text { acy }\end{array}$ & $\begin{array}{l}\text { Sensiti } \\
\text { vity }\end{array}$ & $\begin{array}{l}\text { Specif } \\
\text { icity }\end{array}$ & FPR & $\begin{array}{l}\text { F1 } \\
\text { Score }\end{array}$ & $\begin{array}{l}\text { Comput } \\
\text { ational } \\
\text { Time }\end{array}$ \\
\hline $\begin{array}{l}\text { Alex } \\
\text { Net }\end{array}$ & $\begin{array}{l}0.9905 \\
\pm \\
0.0054\end{array}$ & $\begin{array}{l}0.9905 \\
\pm \\
0.0108\end{array}$ & $\begin{array}{l}0.9995 \\
\pm \\
0.0026\end{array}$ & $\begin{array}{l}0.0005 \\
\pm \\
0.0026\end{array}$ & $\begin{array}{l}0.9950 \\
\pm \\
0.0054\end{array}$ & $\begin{array}{l}28.4800 \\
\pm 0.8819\end{array}$ & $\begin{array}{l}0.6981 \\
\pm 0.07 \\
24\end{array}$ & $\begin{array}{l}0.6229 \\
\pm 0.25 \\
68\end{array}$ & $\begin{array}{l}0.7734 \\
\pm 0.20 \\
63\end{array}$ & $\begin{array}{l}0.2266 \\
\pm 0.20 \\
63\end{array}$ & $\begin{array}{l}0.6431 \\
\pm 0.16 \\
63\end{array}$ & $\begin{array}{l}20.008 \\
7 \pm 2.04 \\
58\end{array}$ \\
\hline $\begin{array}{l}\text { Mobil } \\
\text { eNet } \\
\text { V2 }\end{array}$ & $\begin{array}{l}0.9957 \\
\pm 0.005 \\
5\end{array}$ & $\begin{array}{l}0.9914 \\
\pm 0.011 \\
0\end{array}$ & $\begin{array}{l}1.0000 \\
\pm 0.000 \\
0\end{array}$ & $\begin{array}{l}0.0000 \\
\pm 0.000\end{array}$ & $\begin{array}{l}0.9957 \\
\pm 0.005 \\
6\end{array}$ & $\begin{array}{l}38.3397 \\
\pm 0.5983\end{array}$ & $\begin{array}{l}0.7503 \\
\pm 0.02 \\
99\end{array}$ & $\begin{array}{l}0.7310 \\
\pm 0.10 \\
54\end{array}$ & $\begin{array}{l}0.7716 \\
\pm 0.06 \\
11\end{array}$ & $\begin{array}{l}0.2304 \\
\pm 0.06 \\
11\end{array}$ & $\begin{array}{l}0.7430 \\
\pm 0.03 \\
83\end{array}$ & $\begin{array}{l}35.503 \\
3 \pm 0.35 \\
11\end{array}$ \\
\hline $\begin{array}{l}\text { Shuffl } \\
\text { eNet }\end{array}$ & $\begin{array}{l}0.9933 \\
\pm 0.006 \\
5\end{array}$ & $\begin{array}{l}0.9867 \\
\pm 0.013 \\
0\end{array}$ & $\begin{array}{l}1.0000 \\
\pm 0.000 \\
0\end{array}$ & $\begin{array}{l}0.0000 \\
\pm 0.000\end{array}$ & $\begin{array}{l}0.9932 \\
\pm 0.006 \\
6\end{array}$ & $\begin{array}{l}32.2203 \\
\pm 9.3669\end{array}$ & $\begin{array}{l}0.7527 \\
\pm 0.06 \\
18\end{array}$ & $\begin{array}{l}0.6952 \\
\pm 0.19 \\
84\end{array}$ & $\begin{array}{l}0.8101 \\
\pm 0.13 \\
73\end{array}$ & $\begin{array}{l}0.1899 \\
\pm 0.13 \\
73\end{array}$ & $\begin{array}{l}0.7212 \\
\pm 0.12 \\
79\end{array}$ & $\begin{array}{l}25.701 \\
0 \pm 1.81 \\
24\end{array}$ \\
\hline $\begin{array}{l}\text { Dense } \\
\text { net20 } \\
1\end{array}$ & $\begin{array}{l}0.9964 \\
3 \pm 0.00 \\
487\end{array}$ & $\begin{array}{l}0.9928 \\
6 \pm 0.00 \\
975\end{array}$ & $\begin{array}{l}1.0000 \\
\pm 0.000 \\
00\end{array}$ & $\begin{array}{l}0.0000 \\
0 \pm 0.00 \\
00\end{array}$ & $\begin{array}{l}0.9963 \\
9 \pm 0.00 \\
493\end{array}$ & $\begin{array}{l}131.097 \\
10+8.28 \\
73\end{array}$ & $\begin{array}{l}0.7833 \\
\pm 0.04 \\
27\end{array}$ & $\begin{array}{l}0.7476 \\
\pm 0.13 \\
96\end{array}$ & $\begin{array}{l}0.8899 \\
\pm 0.15 \\
81 \\
\end{array}$ & $\begin{array}{l}0.1810 \\
\pm 0.15 \\
81\end{array}$ & $\begin{array}{l}0.771 \\
\pm 0.05 \\
37 \\
\end{array}$ & $\begin{array}{l}151.06 \\
65 \pm 6.5 \\
647\end{array}$ \\
\hline $\begin{array}{l}\text { Goggl } \\
\text { eNet }\end{array}$ & $\begin{array}{l}0.9957 \\
\pm 0.004 \\
0\end{array}$ & $\begin{array}{l}0.914 \pm \\
0.0080\end{array}$ & $\begin{array}{l}1.0000 \\
\pm 0.000 \\
0\end{array}$ & $\begin{array}{l}0.0000 \\
\pm 0.000 \\
0\end{array}$ & $\begin{array}{l}0.9957 \\
\pm 0.008 \\
0\end{array}$ & $\begin{array}{l}28.7687 \\
\pm 0.2670\end{array}$ & $\begin{array}{l}0.7233 \\
\pm 0.05 \\
43\end{array}$ & $\begin{array}{l}0.7357 \\
\pm 0.18 \\
54\end{array}$ & $\begin{array}{l}0.7110 \\
\pm 0.18 \\
94\end{array}$ & $\begin{array}{l}0.2890 \\
\pm 0.18 \\
94\end{array}$ & $\begin{array}{l}0.7167 \\
\pm 0.08 \\
99 \\
\end{array}$ & $\begin{array}{l}25.185 \\
2 \pm 0.32 \\
40\end{array}$ \\
\hline $\begin{array}{l}\text { Incept } \\
\text { ion } \\
\text { Resne } \\
\text { t V2 } \\
\end{array}$ & $\begin{array}{l}0.9943 \\
\pm 0.007 \\
1\end{array}$ & $\begin{array}{l}0.9886 \\
\pm 0.014 \\
2\end{array}$ & $\begin{array}{l}1.0000 \\
\pm 0.000 \\
0\end{array}$ & $\begin{array}{l}0.0000 \\
\pm 0.000 \\
0\end{array}$ & $\begin{array}{l}0.9942 \\
\pm 0.007 \\
3\end{array}$ & $\begin{array}{l}171.590 \\
6 \pm 2.960 \\
4\end{array}$ & $\begin{array}{l}0.7529 \\
\pm 0.04 \\
88\end{array}$ & $\begin{array}{l}0.7471 \\
\pm 0.14 \\
82\end{array}$ & $\begin{array}{l}0.7586 \\
\pm 0.16 \\
67\end{array}$ & $\begin{array}{l}0.2414 \\
\pm 0.16 \\
67\end{array}$ & $\begin{array}{l}0.7456 \\
\pm 0.07 \\
50\end{array}$ & $\begin{array}{l}175.93 \\
33 \pm 2.5 \\
783\end{array}$ \\
\hline $\begin{array}{l}\text { Incept } \\
\text { ionV3 }\end{array}$ & $\begin{array}{l}0.9944 \\
\pm 0.006 \\
9\end{array}$ & $\begin{array}{l}0.9886 \\
\pm 0.013 \\
7\end{array}$ & $\begin{array}{l}1.0000 \\
\pm 0.000 \\
0\end{array}$ & $\begin{array}{l}0.0000 \\
\pm 0.000 \\
0\end{array}$ & $\begin{array}{l}0.9942 \\
\pm 0.007 \\
0\end{array}$ & $\begin{array}{l}79.4426 \\
\pm 3.8411\end{array}$ & $\begin{array}{l}0.7442 \\
\pm 0.04 \\
62\end{array}$ & $\begin{array}{l}0.7838 \\
\pm 0.11 \\
06\end{array}$ & $\begin{array}{l}0.7046 \\
\pm 0.14 \\
47\end{array}$ & $\begin{array}{l}0.2954 \\
\pm 0.14 \\
47\end{array}$ & $\begin{array}{l}0.7524 \\
\pm 0.04 \\
67 \\
\end{array}$ & $\begin{array}{l}77.863 \\
7 \pm 0.56 \\
11\end{array}$ \\
\hline $\begin{array}{l}\text { Resne } \\
\text { t18 }\end{array}$ & $\begin{array}{l}0.9959 \\
\pm 0.004 \\
8\end{array}$ & $\begin{array}{l}0.9928 \\
\pm 0.007 \\
2\end{array}$ & $\begin{array}{l}0.9990 \\
\pm 0.005 \\
2\end{array}$ & $\begin{array}{l}0.0009 \\
\pm 0.005 \\
2\end{array}$ & $\begin{array}{l}0.9959 \\
\pm 0.004 \\
8\end{array}$ & $\begin{array}{l}31.6206 \\
\pm 9.9852\end{array}$ & $\begin{array}{l}0.7394 \\
\pm 0.06 \\
19 \\
\end{array}$ & $\begin{array}{l}0.6919 \\
\pm 0.19 \\
77\end{array}$ & $\begin{array}{l}0.7869 \\
\pm 0.19 \\
66 \\
\end{array}$ & $\begin{array}{l}0.2130 \\
\pm 0.19 \\
66\end{array}$ & $\begin{array}{l}0.7130 \\
\pm 0.10 \\
62 \\
\end{array}$ & $\begin{array}{l}26.560 \\
3 \pm 0.17 \\
77\end{array}$ \\
\hline $\begin{array}{l}\text { Resne } \\
\text { t50 }\end{array}$ & $\begin{array}{l}0.9967 \\
\pm 0.005 \\
5\end{array}$ & $\begin{array}{l}0.9933 \\
\pm 0.011 \\
1\end{array}$ & $\begin{array}{l}1.0000 \\
\pm 0.000 \\
0\end{array}$ & $\begin{array}{l}0.0000 \\
\pm 0.000 \\
0\end{array}$ & $\begin{array}{l}0.9966 \\
\pm 0.005 \\
6\end{array}$ & $\begin{array}{l}31.6802 \\
\pm 2.2659\end{array}$ & $\begin{array}{l}0.7830 \\
\pm 0.06 \\
98\end{array}$ & $\begin{array}{l}0.7390 \\
\pm 0.18 \\
50\end{array}$ & $\begin{array}{l}0.8270 \\
\pm 0.17 \\
51\end{array}$ & $\begin{array}{l}0.1730 \\
\pm 0.17 \\
51\end{array}$ & $\begin{array}{l}0.7625 \\
\pm 0.11 \\
06\end{array}$ & $\begin{array}{l}53.443 \\
9 \pm 11.1 \\
508\end{array}$ \\
\hline $\begin{array}{l}\text { Resne } \\
\text { t101 }\end{array}$ & $\begin{array}{l}0.9907 \\
\pm 0.007 \\
3\end{array}$ & $\begin{array}{l}0.9852 \\
\pm 0.010 \\
9\end{array}$ & $\begin{array}{l}0.9962 \\
\pm 0.009 \\
9\end{array}$ & $\begin{array}{l}0.0038 \\
\pm 0.009 \\
9\end{array}$ & $\begin{array}{l}0.9907 \\
\pm 0.007 \\
3\end{array}$ & $\begin{array}{l}77.5618 \\
\pm 0.0146\end{array}$ & $\begin{array}{l}0.7676 \\
\pm 0.06 \\
40\end{array}$ & $\begin{array}{l}0.7381 \\
\pm 0.18 \\
23\end{array}$ & $\begin{array}{l}0.7970 \\
\pm 0.15 \\
90\end{array}$ & $\begin{array}{l}0.2030 \\
\pm 0.15 \\
90\end{array}$ & $\begin{array}{l}0.7494 \\
\pm 0.10 \\
64 \\
\end{array}$ & $\begin{array}{l}79.358 \\
4 \pm 1.88 \\
72\end{array}$ \\
\hline $\begin{array}{l}\text { VGG } \\
16\end{array}$ & $\begin{array}{l}0.9945 \\
\pm 0.005 \\
5\end{array}$ & $\begin{array}{l}0.9905 \\
\pm 0.009 \\
4\end{array}$ & $\begin{array}{l}0.9986 \\
\pm 0.007 \\
8 \\
\end{array}$ & $\begin{array}{l}0.0014 \\
\pm 0.007 \\
8\end{array}$ & $\begin{array}{l}0.9945 \\
\pm 0.005 \\
5\end{array}$ & $\begin{array}{l}95.3727 \\
\pm 2.5518\end{array}$ & $\begin{array}{l}0.8135 \\
\pm 0.05 \\
04\end{array}$ & $\begin{array}{l}0.7695 \\
\pm 0.13 \\
25 \\
\end{array}$ & $\begin{array}{l}0.8574 \\
\pm 0.08 \\
33 \\
\end{array}$ & $\begin{array}{l}0.1426 \\
\pm 0.08 \\
33\end{array}$ & $\begin{array}{l}0.7991 \\
\pm 0.07 \\
87 \\
\end{array}$ & $\begin{array}{l}95.468 \\
7 \pm 1.24 \\
79\end{array}$ \\
\hline $\begin{array}{l}\text { VGG } \\
19\end{array}$ & $\begin{array}{l}0.9981 \\
\pm 0.003 \\
7\end{array}$ & $\begin{array}{l}0.9962 \\
\pm 0.007 \\
4\end{array}$ & $\begin{array}{l}1.0000 \\
\pm 0.000 \\
0\end{array}$ & $\begin{array}{l}0.0000 \\
\pm 0.000 \\
0\end{array}$ & $\begin{array}{l}0.9981 \\
\pm 0.003 \\
8\end{array}$ & $\begin{array}{l}111.856 \\
9 \pm 1.256 \\
9\end{array}$ & $\begin{array}{l}0.8128 \\
\pm 0.03 \\
83\end{array}$ & $\begin{array}{l}0.8029 \\
\pm 0.13 \\
49\end{array}$ & $\begin{array}{l}0.8228 \\
\pm 0.12 \\
92\end{array}$ & $\begin{array}{l}0.1772 \\
\pm 0.12 \\
92\end{array}$ & $\begin{array}{l}0.8072 \\
\pm 0.05 \\
14\end{array}$ & $\begin{array}{l}115.91 \\
11 \pm 1.5 \\
280\end{array}$ \\
\hline $\begin{array}{l}X \\
\text { ceptio } \\
n\end{array}$ & $\begin{array}{l}0.9931 \\
\pm 0.006 \\
6\end{array}$ & $\begin{array}{l}0.9862 \\
\pm 0.013 \\
3\end{array}$ & $\begin{array}{l}1.0000 \\
\pm 0.000 \\
0\end{array}$ & $\begin{array}{l}0.0000 \\
\pm 0.000 \\
0\end{array}$ & $\begin{array}{l}0.9930 \\
\pm 0.006 \\
7\end{array}$ & $\begin{array}{l}105.818 \\
0 \pm 0.013 \\
3\end{array}$ & $\begin{array}{l}0.7527 \\
\pm 0.04 \\
80\end{array}$ & $\begin{array}{l}0.7433 \\
\pm 0.15 \\
65\end{array}$ & $\begin{array}{l}0.7620 \\
\pm 0.15 \\
14\end{array}$ & $\begin{array}{l}0.2380 \\
\pm 0.15 \\
14\end{array}$ & $\begin{array}{l}0.7429 \\
\pm 0.08 \\
00\end{array}$ & $\begin{array}{l}110.51 \\
26 \pm 4.6 \\
434\end{array}$ \\
\hline
\end{tabular}


It is observed from Table 2; each classification model performed better in case of Xray images compared to CT scan image. In some classification models, no doubt the computational time is more in case of X-ray image compared to CT scan image but, the other evaluation indicators like accuracy, sensitivity, specificity and F1score have better value. Again, the FPR is always less in case of X-ray image than the CT scan image in all classification model. The accuracy of 13 classification models based on deep feature with SVM using Xray and CT scan images are illustrated in Figure 2.

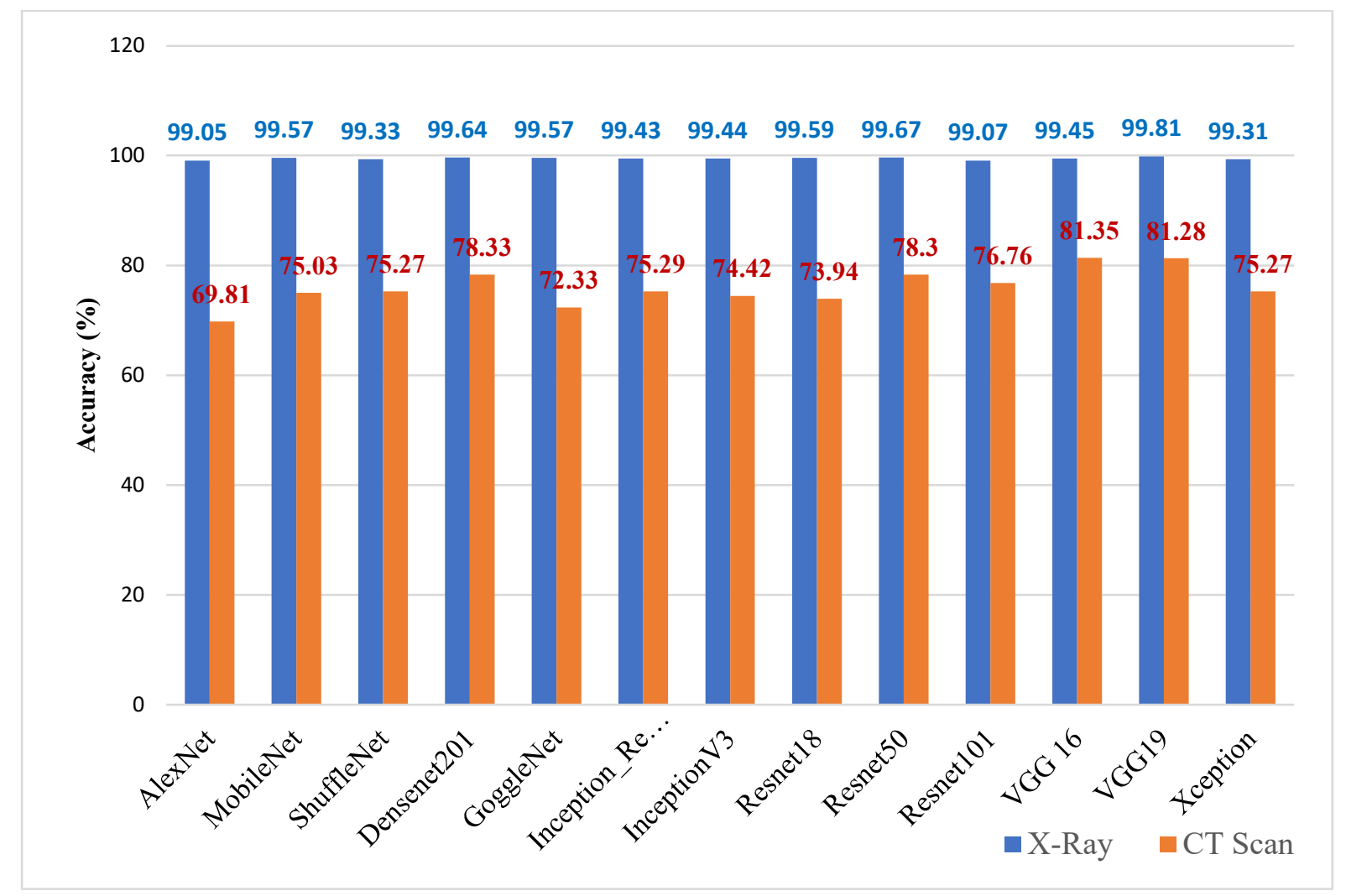

Figure 2 Accuracy in the percentage of classification models based on Deep Feature with SVM approach using Xray and CT scan image.

\subsection{Transfer Learning Approach}

The transfer learning approach is a subset of machine learning that utilise pre-collected knowledge from the established model. This section deals with the screening of COVID19 in the transfer learning approach. In this approach 13 pre-trained network such as alexnet, googlenet, vgg16, vgg19, densenet201, resnet18, resnet50, resnet101, inceptionv3, inceptionresnetv2, xception, mobilenetv2 and shufflenet are evaluated. The classification model in transfer learning approach is illustrated in Figure 3. The evaluation indicators of 13 models are recorded in Table 3. 


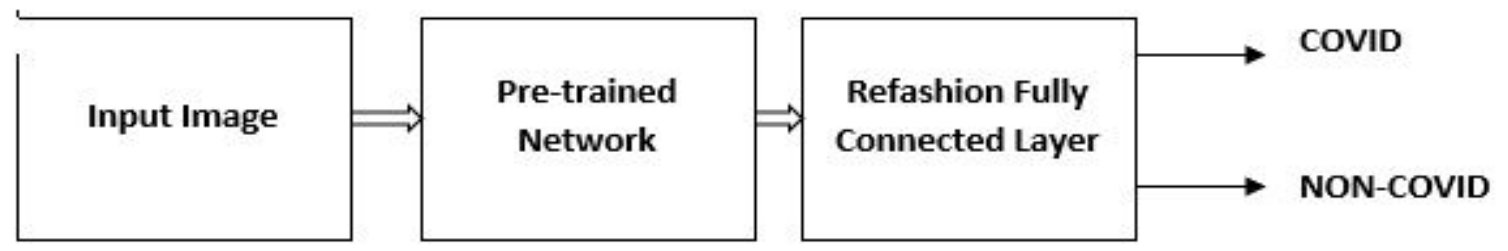

Figure 3. Transfer Learning Approach for Screening of COVID19.

Table 3. Comparative Results of Xray and CT Scan Images in Transfer Learning Approach.

\begin{tabular}{|c|c|c|c|c|c|c|c|c|c|c|c|c|}
\hline \multirow{2}{*}{$\begin{array}{l}\text { Pre- } \\
\text { Traine } \\
\mathrm{d} \\
\text { Model }\end{array}$} & \multicolumn{6}{|c|}{ Xray Image } & \multicolumn{6}{|c|}{ CT Scan Image } \\
\hline & $\begin{array}{l}\text { Accura } \\
\text { cy }\end{array}$ & $\begin{array}{l}\text { Sensiti } \\
\text { vity }\end{array}$ & $\begin{array}{l}\text { Specifi } \\
\text { city }\end{array}$ & FPR & $\begin{array}{l}\text { F1 } \\
\text { Score }\end{array}$ & $\begin{array}{l}\text { Compu } \\
\text { tational } \\
\text { Time }\end{array}$ & $\begin{array}{l}\text { Accur } \\
\text { acy }\end{array}$ & $\begin{array}{l}\text { Sensiti } \\
\text { vity }\end{array}$ & $\begin{array}{l}\text { Specifi } \\
\text { city }\end{array}$ & FPR & $\begin{array}{l}\text { F1 } \\
\text { Score }\end{array}$ & $\begin{array}{l}\text { Comp } \\
\text { utation } \\
\text { al } \\
\text { Time }\end{array}$ \\
\hline $\begin{array}{l}\text { AlexN } \\
\text { et }\end{array}$ & $\begin{array}{l}0.9840 \\
\pm 0.014 \\
0\end{array}$ & $\begin{array}{l}0.9805 \\
\pm 0.021 \\
7\end{array}$ & $\begin{array}{l}0.9878 \\
\pm 0.017 \\
1\end{array}$ & $\begin{array}{l}0.0124 \\
\pm 0.017 \\
1\end{array}$ & $\begin{array}{l}0.9839 \\
\pm 0.014 \\
3\end{array}$ & $\begin{array}{l}65.046 \\
1 \pm 7.31 \\
75\end{array}$ & $\begin{array}{l}0.621 \\
1 \pm 0.0 \\
627\end{array}$ & $\begin{array}{l}0.3695 \\
\pm 0.075 \\
8\end{array}$ & $\begin{array}{l}0.6108 \\
\pm 0.064 \\
4\end{array}$ & $\begin{array}{l}0.3635 \\
\pm 0.0 .0 \\
758\end{array}$ & $\begin{array}{l}0.6108 \\
\pm 0.064 \\
4\end{array}$ & $\begin{array}{l}36.873 \\
1 \pm 4.78 \\
13\end{array}$ \\
\hline $\begin{array}{l}\text { Mobile } \\
\text { Net V2 }\end{array}$ & $\begin{array}{l}0.9864 \\
\pm 0.013 \\
2\end{array}$ & $\begin{array}{l}0.9838 \\
\pm 0.015 \\
8\end{array}$ & $\begin{array}{l}0.9890 \\
\pm 0.018 \\
6\end{array}$ & $\begin{array}{l}0.0110 \\
\pm 0.018 \\
6\end{array}$ & $\begin{array}{l}0.9864 \\
\pm 0.013 \\
2\end{array}$ & $\begin{array}{l}66.209 \\
8 \pm 8.65 \\
27\end{array}$ & $\begin{array}{l}0.642 \\
5 \pm 0.0 \\
433\end{array}$ & $\begin{array}{l}0.6386 \\
\pm 0.062 \\
2\end{array}$ & $\begin{array}{l}0.6462 \\
\pm 0.066 \\
1\end{array}$ & $\begin{array}{l}0.3538 \\
\pm 0.066 \\
1\end{array}$ & $\begin{array}{l}0.6403 \\
\pm 0.045 \\
8\end{array}$ & $\begin{array}{l}39.016 \\
2 \pm 5.28 \\
88\end{array}$ \\
\hline $\begin{array}{l}\text { Shuffle } \\
\text { Net }\end{array}$ & $\begin{array}{l}0.9848 \\
\pm 0.011 \\
8\end{array}$ & $\begin{array}{l}0.9757 \\
\pm 0.020 \\
6\end{array}$ & $\begin{array}{l}0.9938 \\
\pm 0.012 \\
3\end{array}$ & $\begin{array}{l}0.0062 \\
\pm 0.012 \\
3\end{array}$ & $\begin{array}{l}0.9846 \\
\pm 0.012 \\
0\end{array}$ & $\begin{array}{l}65.838 \\
9 \pm 8.12 \\
43\end{array}$ & $\begin{array}{l}0.642 \\
1 \pm 0.0 \\
412 \\
\end{array}$ & $\begin{array}{l}0.6410 \\
\pm 0.055 \\
2\end{array}$ & $\begin{array}{l}0.6433 \\
\pm 0.063 \\
6\end{array}$ & $\begin{array}{l}0.3567 \\
\pm 0.063 \\
6\end{array}$ & $\begin{array}{l}0.6413 \\
\pm 0.042 \\
9\end{array}$ & $\begin{array}{l}39.195 \\
4 \pm 7.73 \\
20\end{array}$ \\
\hline $\begin{array}{l}\text { Densen } \\
\text { et201 }\end{array}$ & $\begin{array}{l}0.9857 \\
\pm 0.009 \\
7\end{array}$ & $\begin{array}{l}0.9810 \\
\pm 0.012 \\
1\end{array}$ & $\begin{array}{l}0.9905 \\
\pm 0.014 \\
7\end{array}$ & $\begin{array}{l}0.0102 \\
\pm 0.014 \\
7\end{array}$ & $\begin{array}{l}0.9857 \\
\pm 0.009 \\
7\end{array}$ & $\begin{array}{l}76.584 \\
8 \pm 9.01 \\
50\end{array}$ & $\begin{array}{l}0.633 \\
4 \pm 0.0 \\
563\end{array}$ & $\begin{array}{l}0.6186 \\
\pm 0.074 \\
5\end{array}$ & $\begin{array}{l}0.6481 \\
\pm 0.075 \\
8\end{array}$ & $\begin{array}{l}0.3519 \\
\pm 0.075 \\
7\end{array}$ & $\begin{array}{l}0.6269 \\
3 \pm 0.05 \\
94\end{array}$ & $\begin{array}{l}50.936 \\
4 \pm 6.31 \\
84\end{array}$ \\
\hline $\begin{array}{l}\text { Goggle } \\
\text { Net }\end{array}$ & $\begin{array}{l}09881 \\
\pm 0.011 \\
0\end{array}$ & $\begin{array}{l}0.9852 \\
\pm 0.014 \\
3\end{array}$ & $\begin{array}{l}0.9910 \\
\pm 0.017 \\
0\end{array}$ & $\begin{array}{l}0.0090 \\
\pm 0.017 \\
0\end{array}$ & $\begin{array}{l}0.9881 \\
\pm 0.010 \\
9\end{array}$ & $\begin{array}{l}65.152 \\
5 \pm 10.0 \\
344\end{array}$ & $\begin{array}{l}0.611 \\
7 \pm 0.0 \\
595\end{array}$ & $\begin{array}{l}0.6195 \\
\pm 0.066 \\
4\end{array}$ & $\begin{array}{l}0.6038 \\
\pm 0.081 \\
6\end{array}$ & $\begin{array}{l}0.3962 \\
\pm 0.081 \\
6\end{array}$ & $\begin{array}{l}0.6145 \\
\pm 0.058 \\
0\end{array}$ & $\begin{array}{l}37.823 \\
6 \pm 4.90 \\
68\end{array}$ \\
\hline $\begin{array}{l}\text { Incepti } \\
\text { on } \\
\text { Resnet } \\
\text { V2 }\end{array}$ & $\begin{array}{l}0.9843 \\
\pm 0.010 \\
5\end{array}$ & $\begin{array}{l}0.9819 \\
\pm 0.014 \\
5\end{array}$ & $\begin{array}{l}0.9867 \\
\pm 0.016 \\
7\end{array}$ & $\begin{array}{l}0.0133 \\
\pm 0.016 \\
7\end{array}$ & $\begin{array}{l}0.9843 \\
\pm 0.010 \\
5\end{array}$ & $\begin{array}{l}82.005 \\
8 \pm 9.01 \\
16\end{array}$ & $\begin{array}{l}0.630 \\
5 \pm 0.0 \\
423\end{array}$ & $\begin{array}{l}0.6300 \\
\pm 0.057 \\
6\end{array}$ & $\begin{array}{l}0.6310 \\
\pm 0.045 \\
7\end{array}$ & $\begin{array}{l}0.3690 \\
\pm 0.045 \\
7\end{array}$ & $\begin{array}{l}0.6297 \\
\pm 0.045\end{array}$ & $\begin{array}{l}55.537 \\
7 \pm 4.04 \\
60\end{array}$ \\
\hline $\begin{array}{l}\text { Incepti } \\
\text { onV3 }\end{array}$ & $\begin{array}{l}0.9895 \\
\pm 0.009 \\
3\end{array}$ & $\begin{array}{l}0.9857 \\
\pm 0.014 \\
0\end{array}$ & $\begin{array}{l}0.9933 \\
\pm 0.011 \\
1\end{array}$ & $\begin{array}{l}0.0067 \\
\pm 0.011 \\
1\end{array}$ & $\begin{array}{l}0.9895 \\
\pm 0.009 \\
4\end{array}$ & $\begin{array}{l}80.915 \\
7 \pm 21.0 \\
157\end{array}$ & $\begin{array}{l}0.621 \\
7 \pm 0.0 \\
573\end{array}$ & $\begin{array}{l}0.6143 \\
\pm 0.074 \\
8\end{array}$ & $\begin{array}{l}0.6290 \\
\pm 0.074 \\
1\end{array}$ & $\begin{array}{l}0.3710 \\
\pm 0.074 \\
1\end{array}$ & $\begin{array}{l}0.6180 \\
\pm 0.060 \\
2\end{array}$ & $\begin{array}{l}39.326 \\
0 \pm 4.17 \\
24\end{array}$ \\
\hline $\begin{array}{l}\text { Resnet } \\
18\end{array}$ & & & & & & & & & & & & \\
\hline $\begin{array}{l}\text { Resnet } \\
50\end{array}$ & $\begin{array}{l}0.9819 \\
\pm 0.015 \\
6 \\
\end{array}$ & $\begin{array}{l}0.9786 \\
\pm 0.017 \\
9\end{array}$ & $\begin{array}{l}0.9852 \\
\pm 0.019 \\
3\end{array}$ & $\begin{array}{l}0.0148 \\
\pm 0.019 \\
3\end{array}$ & $\begin{array}{l}0.9819 \\
\pm 0.015 \\
8\end{array}$ & $\begin{array}{l}65.748 \\
1 \pm 9.39 \\
51\end{array}$ & $\begin{array}{l}0.644 \\
3 \pm 0.0 \\
545\end{array}$ & $\begin{array}{l}0.6281 \\
\pm 0.072 \\
0\end{array}$ & $\begin{array}{l}0.6605 \\
\pm 0.061 \\
6\end{array}$ & $\begin{array}{l}0.3395 \\
\pm 0.061 \\
6\end{array}$ & $\begin{array}{l}0.6375 \\
\pm 0.059 \\
4\end{array}$ & $\begin{array}{l}39.073 \\
5 \pm 4.75 \\
73\end{array}$ \\
\hline $\begin{array}{l}\text { Resnet } \\
101\end{array}$ & $\begin{array}{l}0.9869 \\
\pm 0.010 \\
5\end{array}$ & $\begin{array}{l}0.9857 \\
\pm 0.016 \\
8\end{array}$ & $\begin{array}{l}0.9881 \\
\pm 0.013 \\
6\end{array}$ & $\begin{array}{l}0.0119 \\
\pm 0.013 \\
6\end{array}$ & $\begin{array}{l}0.9869 \\
\pm 0.010 \\
6\end{array}$ & $\begin{array}{l}75.171 \\
0 \pm 10.6 \\
601\end{array}$ & $\begin{array}{l}0.612 \\
6 \pm 0.0 \\
648\end{array}$ & $\begin{array}{l}0.5910 \\
\pm 0.078 \\
3\end{array}$ & $\begin{array}{l}0.6343 \\
\pm 0.080 \\
9\end{array}$ & $\begin{array}{l}0.3657 \\
\pm 0.080 \\
9\end{array}$ & $\begin{array}{l}0.6033 \\
\pm 0.068 \\
7\end{array}$ & $\begin{array}{l}39.928 \\
6 \pm 3.81 \\
58\end{array}$ \\
\hline $\begin{array}{l}\text { VGG } \\
16\end{array}$ & $\begin{array}{l}0.9864 \\
\pm 0.010 \\
2\end{array}$ & $\begin{array}{l}0.9848 \\
\pm 0.013 \\
5\end{array}$ & $\begin{array}{l}0.9881 \\
\pm 0.015 \\
5\end{array}$ & $\begin{array}{l}0.0119 \\
\pm 0.015 \\
5\end{array}$ & $\begin{array}{l}0.9864 \\
\pm 0.010 \\
1\end{array}$ & $\begin{array}{l}81.681 \\
4 \pm 21.6 \\
588\end{array}$ & $\begin{array}{l}0.648 \\
3 \pm 0.0 \\
421\end{array}$ & $\begin{array}{l}0.6386 \\
\pm 0.071 \\
5\end{array}$ & $\begin{array}{l}0.6581 \\
\pm 0.048 \\
3\end{array}$ & $\begin{array}{l}0.3419 \\
\pm 0.048 \\
3\end{array}$ & $\begin{array}{l}0.6435 \\
\pm 0.050 \\
5\end{array}$ & $\begin{array}{l}47.332 \\
5 \pm 12.6 \\
429\end{array}$ \\
\hline $\begin{array}{l}\text { VGG1 } \\
9\end{array}$ & $\begin{array}{l}0.9840 \\
\pm 0.010 \\
9\end{array}$ & $\begin{array}{l}0.9781 \\
\pm 0.012 \\
3\end{array}$ & $\begin{array}{l}0.9900 \\
\pm 0.016 \\
4\end{array}$ & $\begin{array}{l}0.0100 \\
\pm 0.016 \\
4\end{array}$ & $\begin{array}{l}0.9840 \\
\pm 0.010 \\
8\end{array}$ & $\begin{array}{l}78.969 \\
4 \pm 13.7 \\
481\end{array}$ & $\begin{array}{l}0.634 \\
8 \pm 0.0 \\
423 \\
\end{array}$ & $\begin{array}{l}0.6171 \\
\pm 0.075 \\
3\end{array}$ & $\begin{array}{l}0.6524 \\
\pm 0.055 \\
7\end{array}$ & $\begin{array}{l}0.3476 \\
\pm 0.055 \\
7\end{array}$ & $\begin{array}{l}0.6266 \\
\pm 0.053 \\
6\end{array}$ & $\begin{array}{l}39.953 \\
9 \pm 4.56 \\
54\end{array}$ \\
\hline $\begin{array}{l}\mathrm{X} \\
\text { ception }\end{array}$ & $\begin{array}{l}0.987 \pm \\
0.0099\end{array}$ & $\begin{array}{l}0.9810 \\
\pm 0.001 \\
65\end{array}$ & $\begin{array}{l}0.9938 \\
\pm 0.008 \\
9\end{array}$ & $\begin{array}{l}0.0062 \\
\pm 0.008 \\
9\end{array}$ & $\begin{array}{l}0.9873 \\
\pm 0.010 \\
0\end{array}$ & $\begin{array}{l}75.828 \\
1 \pm 12.0 \\
832\end{array}$ & $\begin{array}{l}0.616 \\
9 \pm 0.0 \\
612\end{array}$ & $\begin{array}{l}0.6033 \\
\pm 0.075 \\
4\end{array}$ & $\begin{array}{l}0.6305 \\
\pm 0.075 \\
8\end{array}$ & $\begin{array}{l}0.3695 \\
\pm 0.075 \\
8\end{array}$ & $\begin{array}{l}0.6108 \\
\pm 0.064 \\
4\end{array}$ & $\begin{array}{l}36.783 \\
1 \pm 4.78 \\
13\end{array}$ \\
\hline
\end{tabular}

*The results recorded are the mean and std. dev. of 30 independent runs of each classification models.

It is observed from Table 3; the classification model performs well in case of transfer learning approach compared to CT scan image like deep feature extraction method. Performance measurement of all the twelve pre-trained models in terms of accuracy by using the Transfer Learning Approach is shown in Figure 2. The accuracy of each classification model in transfer learning approach using Xray and CT scan image is shown in Figure 4. 


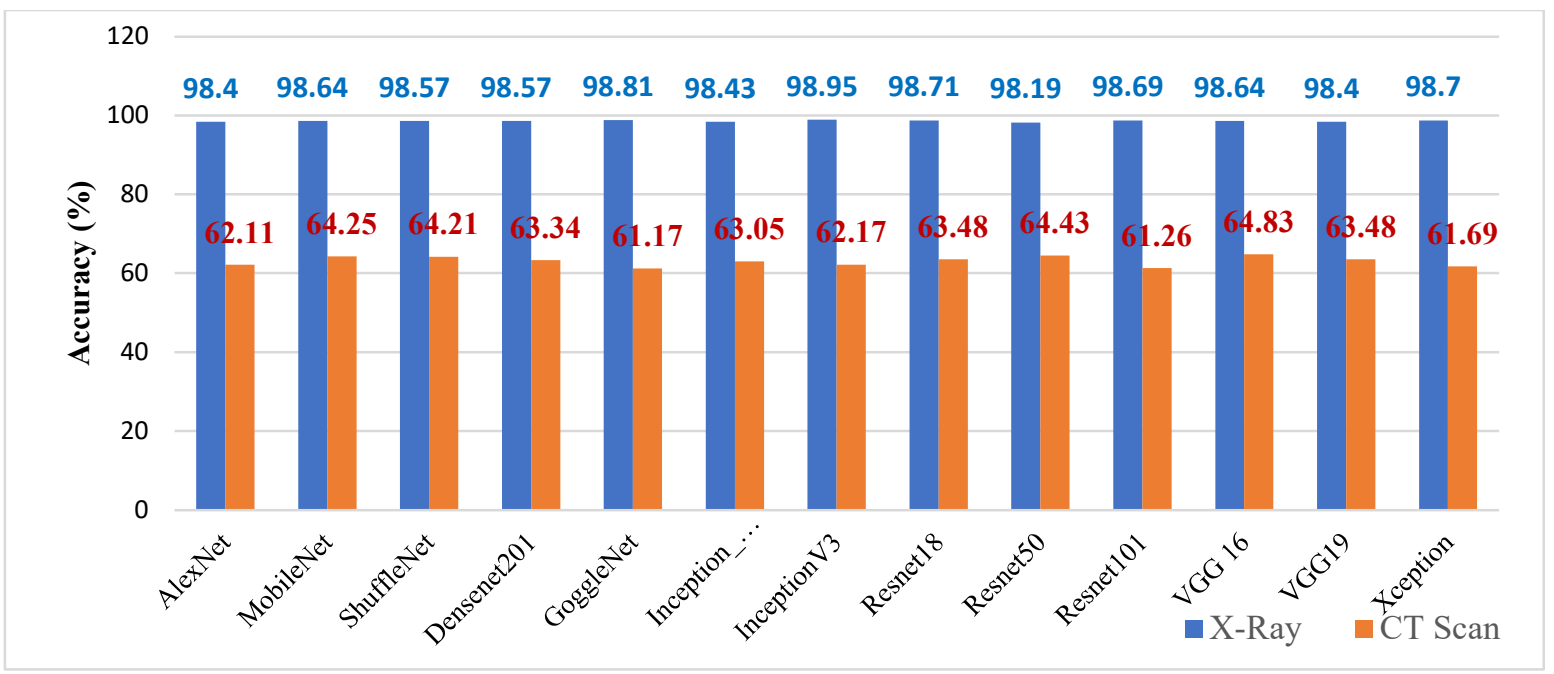

Figure 4 Accuracy in the percentage of classification models based on Transfer Learning approach using Xray and CT scan image.

\subsection{Machine Learning Approach}

The screening of COVID19 using machine learning approach includes HOG, LBP and GLCM feature. Using these features, the classifier such as Naïve bay's, KNN and SVM with their different paradigms are evaluated. The performance of classification models in machine learning approaches is in terms of accuracy and AUC. Table 4 noted the measures of classification models.

Table 4. Comparative Results of Xray and CT Scan Images in Traditional machine learning Approach.

\begin{tabular}{|c|c|c|c|c|c|c|c|c|c|c|c|c|c|}
\hline \multicolumn{2}{|c|}{ Classifier } & \multicolumn{6}{|c|}{ Xray Image } & \multicolumn{6}{|c|}{ CT Scan Image } \\
\hline & & \multicolumn{2}{|c|}{ GLCM } & \multicolumn{2}{|c|}{ HOG } & \multicolumn{2}{|c|}{ LBP } & \multicolumn{2}{|c|}{ GLCM } & \multicolumn{2}{|c|}{ HOG } & \multicolumn{2}{|c|}{ LBP } \\
\hline & & $\begin{array}{c}\text { Accurac } \\
\mathrm{y}\end{array}$ & $\begin{array}{c}\mathrm{AU} \\
\mathrm{C}\end{array}$ & $\begin{array}{c}\text { Accura } \\
\text { cy }\end{array}$ & $\begin{array}{c}\mathrm{AU} \\
\mathrm{C}\end{array}$ & $\begin{array}{c}\text { Accura } \\
\text { cy }\end{array}$ & $\begin{array}{l}\mathrm{AU} \\
\mathrm{C}\end{array}$ & $\begin{array}{c}\text { Accura } \\
\text { cy }\end{array}$ & $\begin{array}{l}\mathrm{AU} \\
\mathrm{C}\end{array}$ & $\begin{array}{c}\text { Accura } \\
\text { cy }\end{array}$ & $\begin{array}{c}\mathrm{AU} \\
\mathrm{C}\end{array}$ & $\begin{array}{c}\text { Accura } \\
\text { cy }\end{array}$ & $\begin{array}{c}\mathrm{AU} \\
\mathrm{C}\end{array}$ \\
\hline $\begin{array}{l}\text { Naïve } \\
\text { Bayes }\end{array}$ & $\begin{array}{l}\text { Kernel } \\
\text { Naïve } \\
\text { Bayes }\end{array}$ & 98.60 & 1.00 & 99.00 & 1.00 & 97.10 & 0.96 & 65.90 & 0.67 & 89.70 & 0.70 & 80.90 & 0.71 \\
\hline \multirow[t]{6}{*}{ SVM } & $\begin{array}{l}\text { Linear } \\
\text { SVM }\end{array}$ & 96.90 & 0.98 & 98.80 & 0.98 & 97.80 & 1.00 & 69.00 & 0.68 & 95.60 & 0.83 & 94.10 & 0.96 \\
\hline & $\begin{array}{l}\text { Quadratic } \\
\text { SVM }\end{array}$ & 97.90 & 0.98 & 100 & 1.00 & 100 & 1.00 & 80.10 & 0.84 & 95.10 & 0.89 & 96.60 & 0.96 \\
\hline & $\begin{array}{l}\text { Cubic } \\
\text { SVM }\end{array}$ & 98.80 & 0.99 & 100 & 1.00 & 100 & 1.00 & 80.00 & 0.84 & 93.60 & 0.86 & 96.60 & 0.95 \\
\hline & $\begin{array}{l}\text { Fine } \\
\text { Gaussian } \\
\text { SVM }\end{array}$ & 97.10 & 1.00 & 97.30 & 1.00 & 98.80 & 1.00 & 79.60 & 0.90 & 94.60 & 0.59 & 94.60 & 0.93 \\
\hline & $\begin{array}{l}\text { Medium } \\
\text { Gaussian } \\
\text { SVM }\end{array}$ & 98.10 & 1.00 & 100 & 1.00 & 98.50 & 1.00 & 77.80 & 0.82 & 95.60 & 0.84 & 94.60 & 0.94 \\
\hline & $\begin{array}{l}\text { Coarse } \\
\text { Gaussian } \\
\text { SVM }\end{array}$ & 96.20 & 0.99 & 96.10 & 0.98 & 94.90 & 0.98 & 63.80 & 0.70 & 94.60 & 0.81 & 94.60 & 0.96 \\
\hline \multirow[t]{6}{*}{ KNN } & Fine $\mathrm{KNN}$ & 99.00 & 0.97 & 100 & 1.00 & 100 & 1.00 & 83.40 & 0.82 & 94.10 & 0.67 & 96.10 & 0.72 \\
\hline & $\begin{array}{l}\text { Medium } \\
\text { KNN }\end{array}$ & 96.00 & 1.00 & 95.90 & 1.00 & 96.80 & 1.00 & 77.80 & 0.81 & 94.60 & 0.83 & 94.60 & 0.87 \\
\hline & $\begin{array}{l}\text { Coarse } \\
\text { KNN }\end{array}$ & 83.60 & 0.99 & 94.6 & 0.63 & 94.6 & 0.97 & 63.60 & 0.67 & 85.20 & 0.98 & 85.20 & 0.87 \\
\hline & $\begin{array}{l}\text { Cosine } \\
\text { KNN }\end{array}$ & 95.20 & 0.99 & 96.40 & 0.99 & 96.80 & 1.00 & 75.40 & 0.81 & 94.60 & 0.85 & 94.60 & 0.83 \\
\hline & $\begin{array}{l}\text { Cubic } \\
\text { KNN }\end{array}$ & 95.20 & 1.00 & 95.60 & 1.00 & 96.80 & 1.00 & 73.60 & 0.80 & 94.60 & 0.86 & 94.60 & 0.86 \\
\hline & $\begin{array}{l}\text { Weighted } \\
\text { KNN }\end{array}$ & 98.80 & 1.00 & 100 & 1.00 & 99.80 & 1.00 & 83.20 & 0.89 & 94.60 & 0.85 & 95.60 & 95.6 \\
\hline
\end{tabular}


For proper comparison of technological benefits among X-ray and CT scan images in readers point of view the accuracy comparison of each classifier with each feature in every paradigm is illustrated in Figure 5, Figure 6 and Figure 7. The accuracy of the different classifier with their paradigms using GLCM feature, HOG feature and LBP feature is illustrated in Figure 5, Figure 6 and Figure 7 respectively. It is observed from Figure 5, Figure 6 and Figure 7, the classification models in traditional machine learning approaches performed well using X-ray images compared to CT scan images.

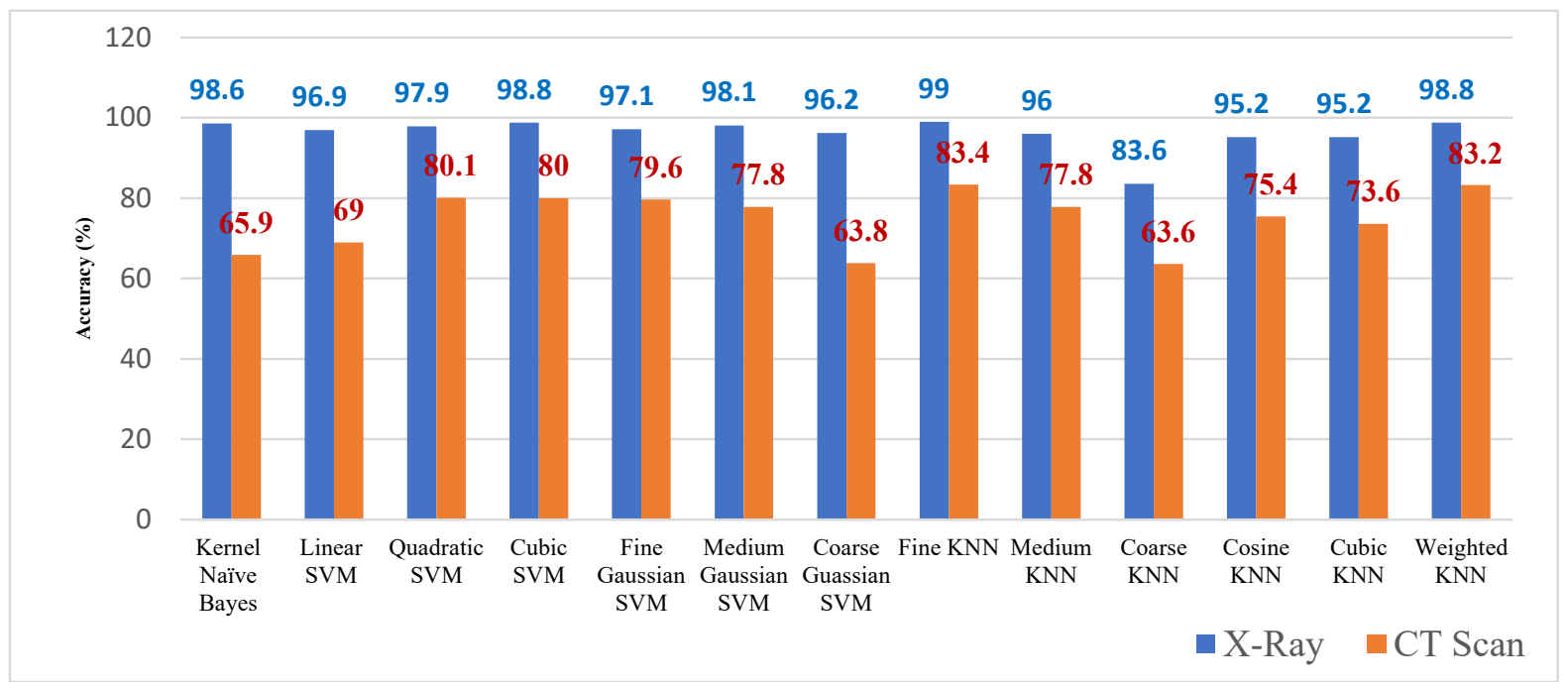

Figure 5 Accuracy of Machine Learning Models using GLCM features.

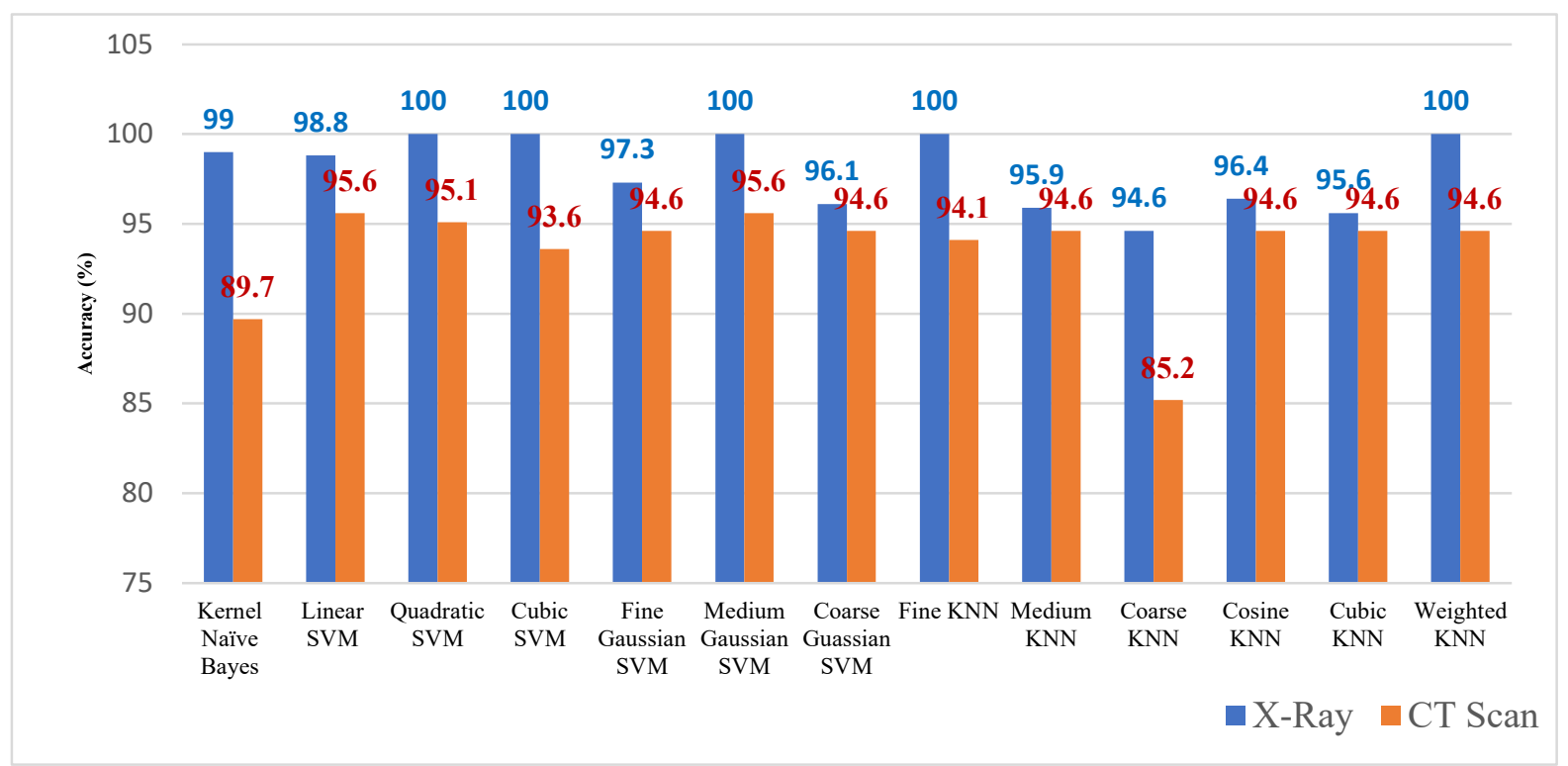

Figure 6 Accuracy of Machine Learning Models using HOG features. 


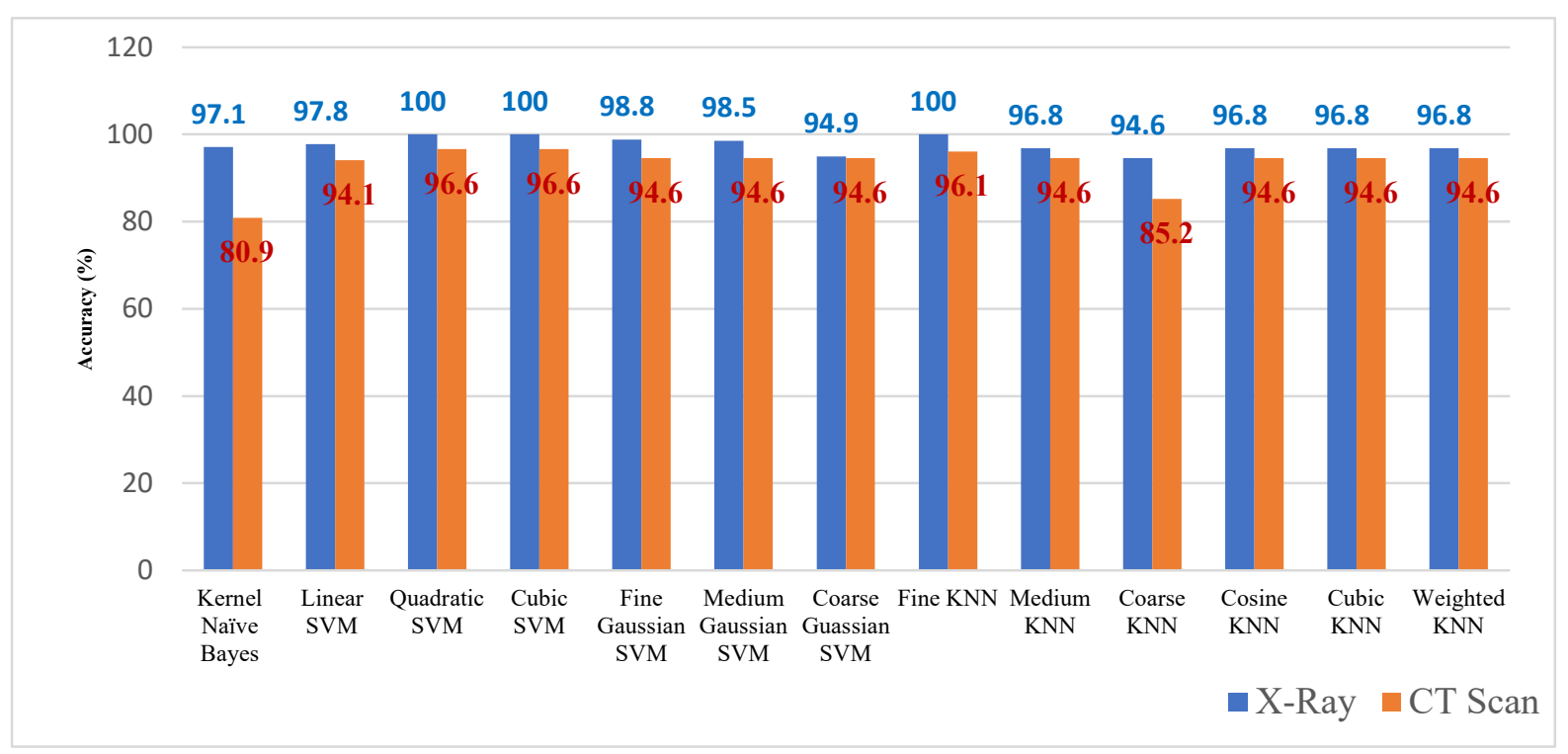

Figure 7 Accuracy of Machine Learning Models using LBP features.

\section{Conclusion}

The comparative study for the screening of COVID19 in deep feature extraction, transfer learning and traditional machine learning is carried out. Here, 65 classification models are evaluated to judge the betterment among the $\mathrm{X}$-ray and CT scan images. It is implicated from the experimentation in the same dimension of the dataset of Xray and CT scan that, the X-ray images are better for computer aid diagnosis. Again, this comparative study provides a clear idea about the limitations and supremacy of X-ray and CT scan image towards the design of computer aid diagnosis system. Also, this comparative study offers additional insight among the research community those are working towards the development of computer aid diagnosis system for screening of COVID19.

\section{References}

[1] https://www.who.int/emergencies/diseases/novel-coronavirus-2019/ situation-reports/.

[2] Toğaçar, M., Ergen, B., Cömert, Z.: COVID-19 detection using deep learning models to exploit Social Mimic Optimization and structured chest X-ray images using fuzzy colour and stacking approaches. Computers in Biology and Medicine. 121, 103805, (2020). https://doi.org/10.1016/i.compbiomed.2020.103805.

[3] Ceylan, Z.: Estimation of COVID-19 prevalence in Italy, Spain, and France. Science of the Total Environment. 729, 138817, (2020). https://doi.org/10.1016/i.scitotenv.2020.138817

[4] Alimadadi, A., Aryal, S., Manandhar, I., Munroe, P.B., Joe, B., Cheng, X.: Artificial intelligence and machine learning to fight COVID-19. Physiological Genomics. 52, 200-202 (2020). https://doi.org/10.1152/physiolgenomics.00029.2020.

[5] Pirouz, B., Shaffiee Haghshenas, S., Shaffiee Haghshenas, S., Piro, P.: Investigating a Serious Challenge in the Sustainable Development Process: Analysis of Confirmed cases of COVID-19 (New Type of Coronavirus) Through a Binary Classification Using Artificial Intelligence and Regression Analysis. Sustainability. 12, 2427 (2020). https://doi.org/10.3390/su12062427. 
[6] Cohen, J.P., Morrison, P., Dao, L., Roth, K., Duong, T.Q., Ghassemi, M.: COVID-19 Image Data Collection: Prospective Predictions Are the Future. (2020).

[7] Kumar Sethy, P., Kumari Behera, S.: Detection of coronavirus Disease (COVID-19) based on Deep Features. (2020). https://doi.org/10.20944/preprints202003.0300.v1.

[8] Narin, A., Kaya, C., Pamuk, Z.: Automatic Detection of Coronavirus Disease (COVID-19) Using X-ray Images and Deep Convolutional Neural Networks. (2020).

[9] Li, Y., Xia, L.: Coronavirus disease 2019 (COVID-19): Role of chest CT in diagnosis and management. American Journal of Roentgenology. 214, 1280-1286 (2020). https://doi.org/10.2214/AJR.20.22954.

[10] Ucar, F., Korkmaz, D.: COVIDiagnosis-Net: Deep Bayes-SqueezeNet based diagnosis of the coronavirus disease 2019 (COVID-19) from X-ray images. Medical Hypotheses. 140, 109761 (2020). https://doi.org/10.1016/j.mehy.2020.109761.

[11] Kang, H., Xia, L., Yan, F., Wan, Z., Shi, F., Yuan, H., Jiang, H., Wu, D., Sui, H., Zhang, C., Shen, D.: Diagnosis of Coronavirus Disease 2019 (COVID-19) with Structured Latent Multi-View Representation Learning. IEEE transactions on medical imaging. (2020). https://doi.org/10.1109/TMI.2020.2992546.

[12] Oh, Y., Park, S., Ye, J.C.: Deep Learning COVID-19 Features on CXR using Limited Training Data Sets. IEEE Transactions on Medical Imaging. 1-1 (2020). https://doi.org/10.1109/tmi.2020.2993291.

[13] Tuncer, T., Dogan, S., Ozyurt, F.: An automated Residual Exemplar Local Binary Pattern and iterative ReliefF based corona detection method using lung X-ray image. Chemometrics and Intelligent Laboratory Systems. 203, 104054 (2020). https://doi.org/10.1016/j.chemolab.2020.104054.

[14] Ozturk, T., Talo, M., Yildirim, E.A., Baloglu, U.B., Yildirim, O., Rajendra Acharya, U.: Automated detection of COVID-19 cases using deep neural networks with X-ray images. Computers in Biology and Medicine. 121, 103792 (2020). https://doi.org/10.1016/i.compbiomed.2020.103792.

[15] Nour, M., Cömert, Z., Polat, K.: A Novel Medical Diagnosis model for COVID-19 infection detection based on Deep Features and Bayesian Optimization. Applied Soft Computing. 106580 (2020). https://doi.org/10.1016/i.asoc.2020.106580.

[16] Panwar, H., Gupta, P. K., Siddiqui, M. K., Morales-Menendez, R., \& Singh, V. (2020). Application of Deep Learning for Fast Detection of COVID-19 in X-Rays using nCOVnet. Chaos, Solitons \& Fractals, 109944. DOI:10.1016/j.chaos.2020.109944

[17] Lalmuanawma, S., Hussain, J., \& Chhakchhuak, L. (2020). Applications of Machine Learning and Artificial Intelligence for Covid-19 (SARS-CoV-2) pandemic: A review. Chaos, Solitons \& Fractals, 110059. DOI:10.1016/j.chaos.2020.110059

[18] Sethy, P. K., Barpanda, N. K., Rath, A. K., \& Behera, S. K. (2020). Deep feature based rice leaf disease identification using support vector machine. Computers and Electronics in Agriculture, 175, 105527. DOI:10.1016/j.compag.2020.105527

\section{Declaration of Competing Interest}

The authors declare that they have no known competing financial interests or personal relationships that could have appeared to influence the work reported in this paper. 